\section{Nuevas políticas de gestión patrimonial en América Latina y el Caribe}

Durante los días 14 al 18 de septiembre, la Universidad Internacional de Andalucía en colaboración con el IAPH, desarrollaron el curso "Políticas patrimoniales en América Latina y El Caribe: nueva institucionalidad", que convocó a expertos de Brasil, México, Chile, Ecuador, Cuba y Argentina que pusieron en común experiencias, reflexiones y proyectos sobre gestión patrimonial y cooperación, proporcionando las claves de cómo repercute en las políticas de gestión patrimonial de toda América Latina la evolución del concepto de patrimonio, a la que se asiste de forma global desde las últimas décadas del siglo pasado.

La evolución del concepto de patrimonio que se produce durante la segunda mitad del siglo $X X$ y, sobre todo, en sus últimas décadas, es el resultado de un profundo debate que queda patente en diferentes cartas y documentos internacionales, tales como Comisión Franceschini (1964), Convención de 1972, Carta de Venecia de 1964, Convención del 2005, etc. Durante mucho tiempo el elemento clave había sido el objeto y fundamentalmente el monumento, así como una visión historicista del patrimonio, con el tiempo cambia la mirada y se comienza a valorar el contexto, se empiezan a considerar el patrimonio natural y cultural como recursos que pueden tener un papel importante en el desarrollo de los distintos países y, en este

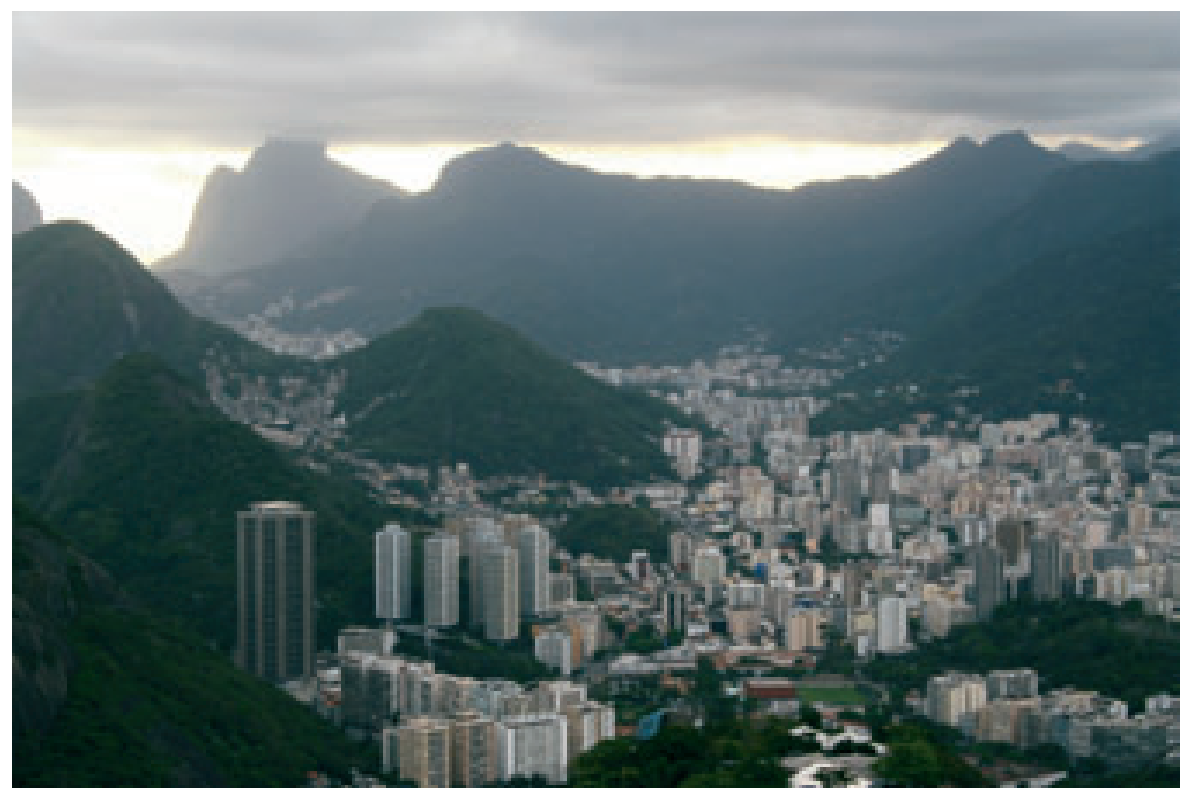

Río de Janeiro, entre morros y agua. Foto: Desiree Vidal
Los países de América Latina y el Caribe comienzan la realización de nuevos inventarios e itinerarios que reflejen su diversidad patrimonial y cultural. Así, en Cuba se hacen inventarios sobre locomotoras a vapor, rutas de esclavos, ruta del cacao, patrimonio azucarero o el patrimonio moderno, y en Argentina se impulsan otros sobre la cultura guarani, la cultura aymara o quechua. En Chile también se avanza bastante en el reconocimiento de la diversidad cultural.

Con la evolución del concepto de patrimonio se empiezan a considerar el patrimonio natural y cultural como recursos importantes en el desarrollo de los pueblos. Se habla de patrimonio y desarrollo y de la necesidad de lograr un equilibrio para que sea sostenible. Las Normas de Quito de 1967, revolucionarias en su día por abordar esa dimensión económica del patrimonio, la participación social, el respeto al patrimonio natural y cultural y la necesidad de incorporar el patrimonio cultural a los planes de desarrollo, el turismo y la rentabilidad, no tuvieron éxito porque se apostó por un modelo de desarrollo europeo sin tener en cuenta que las realidades latinoamericanas eran muy diferentes.

Hay que esperar a la década de los 90 para asistir a un cambio radical en la actuación en los Centros Históricos. Ejemplos importantes son la rehabilitación de la Habana Vieja, la recuperación de Salvador de Bahía en 1992, el Plan de recuperación de la Plaza Mayor de Lima en 1996 o la recuperación del Centro Histórico de Quito. Todos los paises plantean entre sus retos de acción, la apuesta por un desarrollo social y económico que respete los valores culturales y los recursos naturales.

La mayor presencia y consideración que el hombre adquiere en este tipo de patrimonio se hace cada vez más evidente en los últimos años y desde dos ámbitos de acción. En primer lugar la importancia de la participación de los actores implicados, el hombre, la comunidad, el respeto a su identidad para lograr un desarrollo soste- 


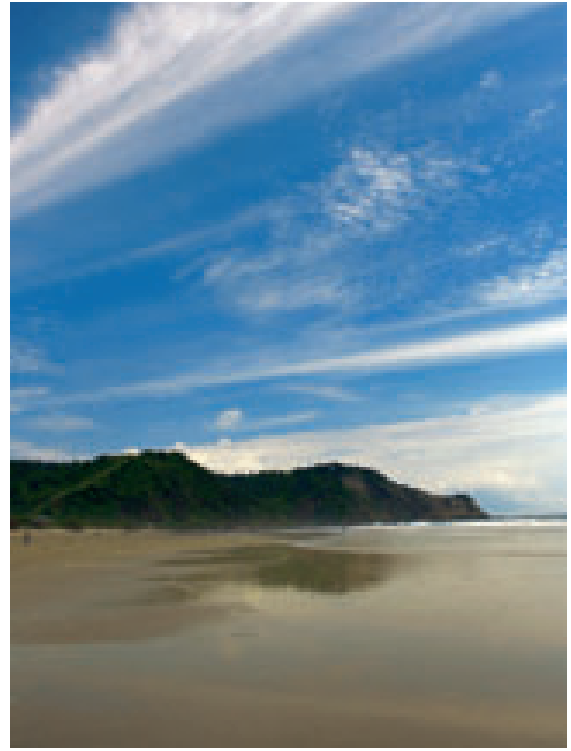

Ruta del Spondylus. Ayampe, Ecuador. Foto: Guillermo Cuesta

nible $y$, en segundo lugar la necesidad de promover acciones de difusión y transferencia del patrimonio y lograr así una mayor sensibilización social, el acercamiento de la comunidad a su patrimonio cultural, considerada como la mejor estrategia para protegerlo y conservarlo.

Para garantizar la protección y conservación del patrimonio cultural, se plantea la necesidad de que los distintos países intervengan, actúen en el patrimonio con el mayor rigor posible, desarrollando estudios científicos y técnicos que perfeccionen los métodos de intervención. Asi lo establece la Convención, y, en este contexto, es manifiesto el interés de la UNESCO en que se disponga de indicadores para evaluar y prever qué va a pasar en las ciudades Patrimonio Mundial en el futuro. Actualmente hay un grupo de técnicos en el IAPH que está trabajando en esta dirección, y se prevé que en cuatro años podrá contarse con un conjunto de indicadores probados en varias ciudades Patrimonio Mundial.

El gran reto de América Latina es gestionar de forma diferente el patrimonio. En los últimos años, en casi todos los paises, se observa una tendencia a realizar cambios institucionales con el objetivo de mejorar la gestión patrimonial. El caso de Ecuador es quizás el más significativo: plan- tea como uno de sus objetivos prioritarios abordar el desarrollo de un nuevo modelo de gestión basado en principios de eficacia y desconcentración. La creación de un Ministerio Coordinador del Patrimonio Natural y Cultural considerado como una de las siete áreas estratégicas del país supone un cambio revolucionario, sobre todo si tenemos en cuenta el nulo interés mostrado por los gobiernos anteriores.

En el caso de Brasil, el cambio estructural del IPHAN, producido en los últimos años, de una estructura funcional a otra temática que da énfasis a los objetos, es sintoma también del esfuerzo realizado en dos frentes: la descentralización y la gestión compartida.

Las herencias compartidas entre los diferentes paises latinoamericanos y el Caribe justifican la necesidad de una mayor interacción, a pesar de la diferencia lingüística en el caso de Brasil. Procesos históricos semejantes y relaciones geográficas, históricas, culturales, etc. están contribuyendo a aumentar la colaboración de forma significativa en los últimos años a través de proyectos conjuntos tales como la formalización de Itinerarios Culturales que trascienden las propias fronteras:

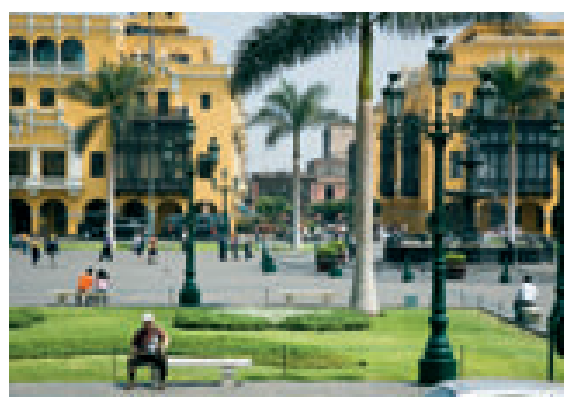

Plaza mayor de Lima. Foto: Pete Lambert

- Desarrollo de Itinerarios Culturales del MERCOSUR como el de Misiones JesuíticoGuaranies o el Itinerario Yerba-Mate, etc.

- Proyecto Regional sobre el Universo Cultural Guaraní, que contempla una base de datos de cada país participante: Argentina, Brasil, Paragüay, Uruguay y Bolivia; y una base de datos común.

- Itinerario o Ruta QHAPACÑAN. Camino andino precolombino. Proyecto en el que participan Ecuador, Perú, Bolivia, Argentina y Chile.

- Ruta SPONDYLUS que tiene como objetivo poner en marcha un Itinerario Cultural que incluya diferentes recursos culturales y turisticos de la costa de Ecuador y de Perú.

La iniciativa de Brasil, en colaboración con la UNESCO de crear un Centro Regional de Formación para la gestión del Patrimonio, a través de MERCOSUR y en colaboración con otros paises de lengua portuguesa, apuesta por una formación generalista y se constituye en un centro especializado y de reflexión sobre el patrimonio. También es destacada la participación de numerosos paises en CRESPIAL, Centro Regional para el Patrimonio Inmaterial de América Latina, ubicado en Perú, con una agenda común para actuaciones compartidas en la Región.

Acciones destacadas de los paises de América Latina y El Caribe

- El esfuerzo de renovación institucional de Ecuador. La existencia de un Ministerio que integra el patrimonio natural y cultural.

- Las experiencias de gestión compartida de Brasil. El proyecto de Santa Catalina tiene como objetivo el desarrollo de un itinerario sobre la inmigración y cuenta con un acuerdo de cooperación técnica entre el IPHAN, los ministerios de Turismo y Desarrollo Agrario, entre otros, para establecer acciones y proyectos conjuntos.

- La búsqueda de un modelo propio de patrimonio y desarrollo sostenible que se muestra en experiencias de Brasil, actuaciones en centros históricos como el de Quito, la Habana Vieja, etc., en las que se observa un gran respeto a la participación de los actores implicados.

- La apuesta por la diversidad cultural, que destaca por ejemplo en Ecuador, Brasil o Chile. - Las diversas experiencias de promoción y sensibilización sobre el patrimonio cultural desarrolladas por Argentina (Buenos Aires ha realizado campañas de sensibilización en escuelas y universidades, visitas a los barrios, recorridos culturales, festivales...), Cuba (labor realizada con escolares en las aulas de los museos locales para que conozcan y valoren la historia y el patrimonio local), etc.

Valle Muñoz Cruz, Centro de Documentación y Estudios del IAPH 